\title{
Clinical study of IUGR cases and correlation of Doppler parameters with perinatal outcome
}

\author{
Deepti D. Sharma*, Kavita C. Chandnani
}

Department of Obstetrics and Gynecology, GMERS Medical College, Gotri, Vadodara, Gujarat, India

Received: 22 September 2016

Revised: 03 November 2016

Accepted: 04 November 2016

\author{
*Correspondence: \\ Dr. Deepti D. Sharma, \\ E-mail: deeptisharma_403@yahoo.co.in
}

Copyright: () the author(s), publisher and licensee Medip Academy. This is an open-access article distributed under the terms of the Creative Commons Attribution Non-Commercial License, which permits unrestricted non-commercial use, distribution, and reproduction in any medium, provided the original work is properly cited.

\begin{abstract}
Background: Early detection and timely intervention in IUGR babies can significantly improve perinatal outcome. Bio physical profile and non stress test have been the common modalities used for foetal surveillance but neither of these is accurate in predicting poor perinatal outcome. With the advent of Doppler, it has become much easier to pick up IUGR cases early and with high reproducibility and efficacy for perinatal outcome. Objectives of this study were 1) to correlate clinical findings in IUGR with colour Doppler and perinatal outcome 2) to study prognostic efficacy of Doppler parameters in early detection of foetal compromise.

Methods: Sixty singleton pregnancies diagnosed to have IUGR beyond 30 weeks gestation were included in the study and were examined and followed clinically as well as with Doppler findings till delivery. Prognostic efficacy (sensitivity, specificity, positive and negative predictive values) of umbilical artery and middle cerebral artery waveforms were studied using percentage.

Results: The sensitivity and specificity of MCA PI $(78 \%, 93 \%)$ as an indicator for extent of foetal compromise in IUGR was highest, though ratios such as MCA PI/UA PI as well as UA SD (>3) were comparably specific predictors for poor perinatal outcome. Absent and reverse end diastolic flow in umbilical artery is associated with definite poor perinatal outcome in terms of mortality. Clinical examination as well as ultrasound biometry has limited accuracy in determining appropriate time of intervention in growth restricted foetuses. Non reactive non stress test was shown to have positive predictive value of only $24 \%$ with false positive rate of $75 \%$.

Conclusions: Colour Doppler study is a simple, quick, non invasive procedure and multiple vessel study has high accuracy among the other tests for ante partum foetal surveillance.
\end{abstract}

Keywords: Middle cerebral artery PI, IUGR, Umbilical artery PI

\section{INTRODUCTION}

The development of a good utero-placental circulation is essential for achievement of a normal pregnancy. Prevention of low birth weight (LBW) is a public health priority in India where, the condition is largely attributed to IUGR. A foetus affected by IUGR forms a subset of cases of Small for Gestational Age (SGA) infants. ${ }^{1}$ New born with birth weight less than $2500 \mathrm{gm}$ are referred to as LBW (low birth weight babies) whereas new born with birth weight less than the 10th percentile for their gestational age and less than $2500 \mathrm{gm}$ are referred to as SGA (small for gestational age). IUGR is defined as a foetus that has failed to achieve a specific and arbitrary anthropometric or weight threshold $\left(<10^{\text {th }}\right.$ percentile) by a specific gestational age due to some pathologic process that inhibits expression of the normal intrinsic growth potential. ${ }^{2}$ SGA is a statistical definition while IUGR is a clinical definition and includes neonates with clinical evidence of malnutrition. It can be symmetric (33\%), asymmetric $(55 \%)$ or mixed $(12 \%)$. Several factors either maternal foetal or placental can lead to IUGR, 
commonest being Placental insufficiency (pre eclampsia chronic HT, DM, renal disease, cardiac disease, anaemia) $75-80 \%$, maternal condition not associated with Placental insufficiency (Severe malnutrition, Smoking, Alcohol ingestion, Hemoglobinopathies) 5\%, Foetal chromosomal abnormality 5\%, Multifactorial foetal abnormalities 2-3 $\%$, Foetal infections $1 \%$. It is associated with an increased risk of perinatal mortality, morbidity, and impaired neurodevelopment. ${ }^{2,3}$ Thus, prediction of risk and correct detection of the compromised IUGR foetus to allow for timely intervention is a main objective of antenatal care. Clinical examination as well as ultrasound parameters helps in early detection of IUGR. The most common methods for evaluating health in foetuses identified as SGA have been the biophysical profile (BPP) and the non-stress test (NST). Unfortunately, neither of these tests is particularly sensitive for predicting poor outcome in IUGR pregnancies. Ultrasound is frequently utilized in antenatal period to assess foetal size through serial biometric measurement, amniotic fluid index and the velocimetry analysis of UA and MCA. ${ }^{4}$ It is here that role of Colour Doppler comes to detect these abnormal vascular resistance patterns. ${ }^{5}$ Foetal umbilical arteries is the mainstay of risk assessment in small and compromised foetuses. ${ }^{6}$ Though, multiple vessel study improves the accuracy of Doppler as a whole and is more reliable and helpful in deciding the mode and timing of intervention. This study was aimed at co relating clinical as well as Doppler findings in IUGR and studying the prognostic efficacy of Doppler parameters to decide time of intervention.

\section{METHODS}

A prospective observational study conducted in Department of Obstetrics and Gynaecology in GMERS Gotri Medical College from Jan 2013- Jan 2014. The study was conducted with 60 antenatal subjects who were selected from the outpatient department and from antenatal ward. All the women had a singleton pregnancy of $>30$ weeks gestation.

\section{Inclusion criteria}

- All pregnant women irrespective of age and parity with high risk factors or IUGR clinically

- Normal foetal anatomy

\section{Exclusion criteria}

- Multiple pregnancy

- Congenital anomalies in baby

\section{Methods of the Study}

The subjects enrolled for the study were followed up from the point of recruitment up to the time of delivery. Screening and diagnosis for IUGR includes: ${ }^{7}$
1. Accurate determination of the gestational age.

2. Abdominal palpation to determine fundal height during each antenatal visit.

3. Ultrasound examination of a suspected SGA foetus.

4. Assessment of foetal well-being when an SGA foetus or IUGR foetus is diagnosed. This includes Doppler studies and cardiotocography monitoring (CTG).

\section{Determination of gestational age}

A dating ultrasound in the first trimester provides the most accurate method to determine gestational age. ${ }^{7}$ If the earliest ultrasound is between 13 and 24 weeks of pregnancy and the last menstrual period (LMP) is certain, with regular menstruation, and there is a difference of less than 10 days between LMP and ultrasound, LMP estimate has fair accuracy. If the LMP is uncertain or irregular menstruation, ultrasound EDD is preferred. ${ }^{8}$

- At each antenatal visit, clinically, weight, increase in fundal height, blood pressure was noted by the same observer.

- Gestational age confirmation was done by LMP if patient was sure (mamta card) or by First trimester ultrasound if available. All patients were evaluated first by grey scale ultrasound. Foetal weight was estimated according to Hadlock's formula. AFI was calculated. FL/AC and $\mathrm{HC} / \mathrm{AC}$ were counted.

- The patients showing signs and symptoms of IUGR were subjected to ultrasonography and Doppler studies of the umbilical artery, uterine artery and the Middle cerebral artery. Ductus venosus flow was studied in only few subjects due to technical difficulties.

- Patients having IUGR were monitored using NST, ultrasonography, and Doppler.

- The pregnancies were followed up and data were collected regarding mode of delivery, gestational age at birth, birth weight, 5-min Apgar score, number of foetal and perinatal deaths and admission to NICU.

- Study of various foetal vessels was performed using pulsed Doppler ultrasound (LOGIQ C5 GE Medical systems) with $3.5 \mathrm{MHz}$ curvilinear probe. The following vessels were studied with the mother in a recumbent position during foetal inactivity and apnoea.

\section{Umbilical Artery (UA) \\ 2. Middle Cerebral Artery (MCA)}

- The above vessels were located in the standard plane.

- The umbilical artery measurements were made from free loop of cord midway between the placental and abdominal wall insertion.

- The middle cerebral artery was located in a transverse plane at the level of the lesser wing of the sphenoid bone with sample gate placed on proximal portion of the vessel. 
- Flow velocity waveforms, the resistance index (R.I), pulsatility index (P.I), systolic/diastolic ratio (S/D) of umbilical artery, middle cerebral artery were noted.

\section{Assessment standards}

- S/D ratio, resistance and pulsatality index of umbilical artery (>2SD), middle cerebral artery $(<5$ th percentile) for the gestational age according to the standard reference values.

- Umbilical artery RI reference was according to Kurmanavicius et al. ${ }^{9}$

- The reference value of umbilical artery P.I., cerebroumbilical ratio is according to Dandolo Gramellini et al 10 and MCA P.I. ratio are according to Giancarlo Mari et al. ${ }^{11}$

Reference values by Rajan et al and Acharya $\mathrm{G}$ et al were taken for Umbilical artery S/D ratio. ${ }^{12,13}$ The ratios examined were considered abnormal when-(1) MCA/UA PI ratio less than 1.08 or less than $2 \mathrm{SD}^{10}{ }^{10}$ (2) MCA/UA S/D ratio less than $1 .^{4}$

\section{Abnormal perinatal outcome parameters}

1. Foetal demise (IUD/Stillbirth).

2. Neonatal death within 30 days.

3. 5 min Apgar score $<7$.

4. Admission to NICU for $>24 \mathrm{hrs}$

5. Neonatal morbidity like hyaline membrane disease, intracranial haemorrhage, early onset septicaemia, hypoglycaemia, or neonatal hyperbilirubinemia.

6. Caesarean section for foetal distress.

7. Meconium stained liquor

\section{Statistical analysis}

All data was analyzed using the Microsoft Excel software. Statistical analysis of data was done after compiling and tabulation of data. The sensitivity, specificity, positive predictive value, percentage of false positive and negative results were calculated and compared with other studies.

\section{RESULTS}

Demographic characteristics of the study population are depicted in Table 1. The age group range was 19-26yrs while mean maternal age was 23 yrs. In our study, Pre eclampsia was the most common cause of IUGR $(50 \%$, $\mathrm{n}=30)$, followed by anaemia $(35 \%, \mathrm{n}=21)$. Out of 30 cases, 16 patients had severe pre eclampsia, severe anaemia was present in 15 cases out of 21, 12 patients had both severe pre eclampsia co existent with severe anaemia. Mean gestational age was 33.4 weeks as some patients needed termination due to severe pre eclampsia while some spontaneously progressed to normal labour. Majority of the patients $(61 \%)$ had to undergo caesarean section, most common indication being foetal distress or meconium stained liquor and severe pre eclampsia with severe oligohydramnios.

Table 1: Maternal characteristics of study population.

\begin{tabular}{|ll|}
\hline Maternal characteristics & N $\%$ \\
\hline Parity & \\
\hline Primi & $36(60)$ \\
Multi & $24(40)$ \\
\hline & $*($ n more than 60 as \\
Pregnancy complications & $\begin{array}{l}\text { some patients had more } \\
\text { than one complication) }\end{array}$ \\
\hline Pre eclampsia & 30 \\
Oligohydramnios & 15 \\
Anemia & 21 \\
Cardiac & 2 \\
Post datism & 4 \\
\hline Mode of delivery & \\
\hline Vaginal & $23(38 \%)$ \\
Lscs & $37(61 \%)$ \\
\hline Indications of cs & \\
\hline Fetal distress/ meconium & \\
stained liquor & $12(32 \%)$ \\
Severe oligo & $6(16 \%)$ \\
Severe pre eclampsia with & $12(32 \%)$ \\
severe oligo & $7(18.9 \%)$ \\
Failed induction & \\
\hline
\end{tabular}

Six patients had either AEDF OR REDF in Doppler study, all these foetuses had poor perinatal outcome. None of the patients in our study had abnormal DV waveforms in Doppler as most of the cases were intervened before that.

Table 2: Perinatal outcome of study population.

\begin{tabular}{|ll|}
\hline Perinatal outcome & N \\
\hline Birth weight(gms) & \\
\hline$>=2500$ & $5(8.3 \%)$ \\
$1500-2499$ & $29(48.3 \%)$ \\
$1001-1500$ & $20(33.3 \%)$ \\
$<1000$ & 6 \\
\hline Live births & 56 \\
\hline Still births & 4 \\
\hline Term babies & $27(45 \%)$ \\
\hline Pre term & $33(55 \%)$ \\
\hline Neonatal death & 3 \\
\hline 5 min apgar $<7$ & 9 \\
\hline Neonatal complications & $16(28.5 \%)$ \\
\hline Admission to nicu & $26(46.4 \%)$ \\
\hline
\end{tabular}

Table 2 shows the perinatal outcome in the study population. $46 \%$ of the live births needed admission in NICU. Three neonatal deaths were observed. Those were the foetuses with absent or reverse umbilical artery diastolic flow. Neonatal hyperbilirubinemia was more common complication while severe adverse outcomes like necrotising enterocolitis and 
intracranial/intraventricular haemorrhage were very rare. These foetuses were given double surface phototherapy and were kept in NICU for at least seven days. It seemed to be more of pre term complication rather than due to IUGR. As the birth weight of most of the babies was in the range $1.5-2.5 \mathrm{~kg}$ the catch up growth was fast and normal. Metabolic complications were less to be observed. We studied diagnostic accuracy of Doppler parameters and fundal height as well as FL/AC ratio on grey scale B mode scan (Table 3).

Table 3: Performance characteristics of different parameters studied.

\begin{tabular}{|lllll|}
\hline $\begin{array}{l}\text { Parameter } \\
\text { assessed }\end{array}$ & Sensitivity & Specificity & PPV & NPV \\
\hline $\begin{array}{l}\text { Fundal } \\
\text { height } \\
\text { difference } \\
(>4 \mathrm{CM})\end{array}$ & $75 \%$ & $66 \%$ & $77 \%$ & $64.1 \%$ \\
\hline $\begin{array}{l}\text { FL/AC } \\
>23.5\end{array}$ & $57 \%$ & $76 \%$ & $77 \%$ & $55 \%$ \\
$<=23.5$ & & & & \\
\hline UA SD ratio & & $61 \%$ & $82 \%$ & $58 \%$ \\
\hline $\begin{array}{l}>95 \\
\text { Percentile } \\
<95\end{array}$ & $60 \%$ & $68.9 \%$ & $62.5 \%$ & $66 \%$ \\
\hline $\begin{array}{l}>3 \\
<3\end{array}$ & $59.3 \%$ & $81 \%$ & $75 \%$ & $66 \%$ \\
\hline UA PI & $65 \%$ & $75 \%$ & $91.6 \%$ & $83 \%$ \\
\hline MCA PI & $78 \%$ & $93.7 \%$ & $86 \%$ & $54 \%$ \\
\hline $\begin{array}{l}\text { MCA } \\
\text { PI/UA PI }\end{array}$ & $54 \%$ & $86.9 \%$ & $65.2 \%$ & $61.2 \%$ \\
\hline $\begin{array}{l}\text { MCA } \\
\text { SD/UA SD }\end{array}$ & $55.5 \%$ & $70.3 \%$ & & \\
\hline
\end{tabular}

A significant lag in fundal height is a 4-cm or greater difference than expected for gestational age. Even carefully performed fundal height measurements are seen to only have 26 to 76 percent sensitivity in predicting IUGR. ${ }^{14}$ In our study the sensitivity and specificity of fundal height measurement were found to be 75 and 66 $\%$, respectively. Ultrasonography B mode is normally the first study done to assess IUGR. It loses its accuracy as the pregnancy advances; however the sensitivity and positive predictive value can be improved if several variables are combined such as estimated foetal weight, head circumference and abdominal circumference. ${ }^{15}$ In all growth-retarded foetuses, the abdominal circumference is the first biometric measure to change. ${ }^{16} \mathrm{We}$ studied FL/AC ratio with a cut off of 23.5 , and found the sensitivity and specificity to be $57 \%$ and $76 \%$ respectively. Benson $\mathrm{CB}$ et al ${ }^{17}$ studied the ratio and found the sensitivity to be $56 \%$ and the specificity $74 \%$ and concluded that the FL/AC ratio, though an ageindependent measure whose mean value differs in normal and IUGR foetuses, is not clinically useful as a predictor of IUGR. Even assuming an IUGR prevalence rate of $10 \%$, the likelihood of IUGR in a foetus with an FL/AC ratio above the cut off is only $19 \%$. Different studies have found $\mathrm{HC} / \mathrm{AC}$ and TCD/AC ratios to be better parameters for predicting asymmetric IUGR. ${ }^{18}$ The ratio of head circumference to abdominal circumference is normally one at 32 to 34 weeks and falls below one after 34 weeks. A ratio of greater than one detects about 85 percent of growth-restricted foetuses. ${ }^{16}$ Sensitivity of abdominal circumference for detecting a birth weight less than the 10th centile ranges from $48 \%$ to $87 \%$, with specificity from $69 \%$ to $85 \%$. $^{19}$

Table 4: Neonatal complications.

\begin{tabular}{|lll|}
\hline Complication & N & $\%$ \\
\hline Intracranialhemorrhage & 1 & 1.7 \\
\hline Neonatal hyperbilirubinemia & 8 & 14 \\
\hline Early onset septicemia & 4 & 7 \\
\hline Hypoxic ischemic encephalopathy & 3 & 5 \\
\hline Necrotising enterocolitis & 0 & 0 \\
\hline
\end{tabular}

Among the Doppler parameters, in our study UA PI and MCA PI were found to be most sensitive indicators for adverse perinatal outcome (65\% and $78 \%$ respectively) while MCA PI was the most specific indicator (93.7\%). In study conducted by Gramellini et al, sensitivity of UA PI was found to be $64 \%$ comparable to our study however there was significant difference in the sensitivity of MCA PI (24\%), though it was most specific indicator $(100 \%)$ in their study also. ${ }^{10}$ The difference may be due to smaller sample size in our study. The study conducted by Lakhar et al had comparable results with our study except MCA PI sensitivity $(41.6 \%$ vs. $78 \%)$ and UA SD ratio $(>3)$ specificity (45.4\% vs. $81 \%$ respectively). ${ }^{5}$ The cerebro placental ratio $(\mathrm{CPR}<1)$ was found to have high specificity and positive predictive value than Umbilical artery $\mathrm{SD}$ ratio. This is due to the reason that SD ratio takes into consideration maximum velocity in systole and minimum velocity in end diastole only whereas Pulsatility index involves mean velocity so that if the diastolic flow is affected the SD ratio will be altered. Non stress test was performed regularly depending upon the Doppler findings, however, the sensitivity of non reactive non stress test was found to be only $55 \%$ with positive predictive value of $25 \%$ and high false positive rates. Thus, alone non stress test does not have high accuracy in predicting poor perinatal outcome but it can be of importance in deciding time of intervention in early foetal compromise evident on Doppler, though Doppler still remains the main study in deciding management.

\section{DISCUSSION}

The high incidence of intrauterine growth retardation (IUGR) in general obstetric population $(\sim 10 \%)$ and its low recognition $(<40 \%)$ together lead to increasing perinatal morbidity and mortality. Clinical examination along with Doppler correlation is a must to pick IUGR babies. Evidence on fundal height assessment is mixed, with some studies reporting it to be a good predictor for IUGR, whereas others fail to find much benefit. ${ }^{20,21}$ 
Table 5: Comparison with other studies.

\begin{tabular}{|c|c|c|c|c|c|c|}
\hline \multicolumn{2}{|c|}{ Parameter assessed } & Author & Sensitivity & Specificity & PPV & NPV \\
\hline \multirow{4}{*}{ UA PI } & $>2 \mathrm{SD}$ & Gramellini et al & $64 \%$ & $70.02 \%$ & $72.7 \%$ & $66.7 \%$ \\
\hline & $>2 \mathrm{SD}$ & Lakhar et al & $50 \%$ & $59 \%$ & $66.6 \%$ & $41.9 \%$ \\
\hline & $>2 \mathrm{SD}$ & Fong & $44.7 \%$ & $86.6 \%$ & $54 \%$ & $86.7 \%$ \\
\hline & $>95^{\mathrm{TH}}$ Percentile & Present study & $65 \%$ & $75 \%$ & $75 \%$ & $66 \%$ \\
\hline \multirow[t]{5}{*}{ MCA PI } & & Gramellini et al & $24 \%$ & $100 \%$ & $100 \%$ & $77.3 \%$ \\
\hline & $<5^{\mathrm{TH}}$ Percentile & Lakhar et al & $41.6 \%$ & $90.9 \%$ & $88.2 \%$ & $48.7 \%$ \\
\hline & $<2 \mathrm{SD}$ & Fong & $72.4 \%$ & $58.1 \%$ & $37.7 \%$ & $85.7 \%$ \\
\hline & & Mishra D et al & $43 \%$ & $80 \%$ & & \\
\hline & $<5^{\mathrm{TH}}$ Percentile & Present study & $78 \%$ & $93.7 \%$ & $91.6 \%$ & $83 \%$ \\
\hline \multirow[t]{4}{*}{ UA SD } & $>2 \mathrm{SD}$ & Strigini & $53 \%$ & $94 \%$ & $40 \%$ & $96 \%$ \\
\hline & $>2 \mathrm{SD}$ & Lakhar & $66.6 \%$ & $45.4 \%$ & $66.6 \%$ & $45.4 \%$ \\
\hline & $>95^{\text {th }}$ percentile & \multirow{2}{*}{ Present study } & $60 \%$ & $68.9 \%$ & $62.5 \%$ & $66 \%$ \\
\hline & $>3$ & & $59.3 \%$ & $81 \%$ & $82 \%$ & $58 \%$ \\
\hline \multirow[t]{4}{*}{ MCA/UA PI } & $<1$ & Gramellini et al & $68 \%$ & $98.4 \%$ & $94.4 \%$ & $88 \%$ \\
\hline & $<1$ & Lakhar et al & $47.2 \%$ & $86.3 \%$ & $85 \%$ & $50 \%$ \\
\hline & $<2 \mathrm{SD}$ & Fong et al & $51.3 \%$ & $80.6 \%$ & $48.1 \%$ & $82.5 \%$ \\
\hline & $<1$ & Present study & $54 \%$ & $86.9 \% \%$ & $86 \%$ & $54 \%$ \\
\hline \multirow[t]{2}{*}{ MCA/UA SD } & $<1$ & Lakhar et al & $55.5 \%$ & $72.7 \%$ & $76.9 \%$ & $50 \%$ \\
\hline & $<1$ & Present study & $55.5 \%$ & $70.3 \%$ & $65.2 \%$ & $61.2 \%$ \\
\hline
\end{tabular}

It seems to be more of surveillance than a screening tool as its strength lies in serial assessment. This reduces the accuracy and increases interob server variation. Measurement and plotting of fundal height on customized growth charts against routine clinical assessment by palpation was found to significantly increase antenatal detection of SGA babies from $29 \%$ to $54 \% .^{22,23}$ Thus, Serial measurement of fundal height and plotting on customized growth charts are recommended by the Royal College of Obstetricians and Gynaecologists guidelines. ${ }^{24}$ However, certain pregnancies are not suitable for primary surveillance by fundal height measurement and require ultrasound biometry instead such as (1) fundal height measurement unsuitable (e.g., due to fibroids, high maternal body mass index, deviated uterus) or Breech / transverse presentation (2) Uncertain dates (3) twins / hydramnios (4) pregnancy considered high risk (e.g., due to previous history of SGA). Placenta-based intrauterine growth restriction (IUGR) is predominantly a vascular disorder. It starts with abnormal tertiary villous vessels and ends with characteristic foetal multi-vessel cardiovascular manifestations. ${ }^{25}$ These effects can be documented with Doppler ultrasound examination of a number of vessels: maternal uterine arteries and the foetal umbilical arteries for the placenta; middle cerebral artery (MCA) for preferential brain perfusion; and precordial veins for the cardiac effects of placental dysfunction. As IUGR worsens, Doppler abnormalities in these vascular territories also deteriorate, suggesting a sequential pattern of disease progression. ${ }^{25}$ This presumed sequence and the anticipation of foetal deterioration forms the basis for Doppler surveillance in IUGR. In normal pregnancy, the three indices; S/D; Pl and Rl decrease with advancing gestation in Umbilical artery. ${ }^{26}$ But in IUGR first there is decreased diastolic flow in the umbilical artery due to increase in the resistance that occurs in small arteries and arterioles of the tertiary villi. This raises the S/D ratio; Pl and $\mathrm{Rl}$ of umbilical artery. As the placental insufficiency worsens, the diastolic flow decreases, then become absent, and later reverses. Yoon et al demonstrated in their study that absent umbilical artery waveform is a strong and independent predictor of adverse perinatal outcome. $^{27}$ Foetal MCA is a low resistance circulation throughout pregnancy. It is highly sensitive to foetal hypoxia which induces redistribution of cardiac output towards foetal brain (brain sparing effect) which leads to increase in diastolic flow with decreased pulsatility index of MCA. As MCA/UA ratio incorporates data not only on placental status but also on foetal response, an abnormal MCA/UA PI Doppler ratio is strongly correlated with worse foetal prognosis. In normal pregnancies the diastolic component in the cerebral arteries is lower than in the umbilical arteries at any gestational age. Therefore, the cerebrovascular resistance remains higher than the placental resistance and the MCA/UA PI is greater than 1. The index becomes less than 1 if the flow distribution is in favour of the brain in pathological pregnancies. In our study the CPR had a high specificity and positive predictive value $(86 \%$ and $86.9 \%)$ similar to Gramellini et al and Lakhar et al, Shahina Bano et al showed $100 \%$ specificity of this parameter. ${ }^{5,10,28}$ Changes in the venous circulation, ductus venosus, are shown to be associated with severely compromised foetus and correlate better with foetal acidosis according to Rizzo et al requiring urgent intervention, though in our study none of the cases reached to that stage. ${ }^{29}$ Our results were also comparable 
with those of K. W. Fong et al, who showed high sensitivity and negative predictive value of MCA PI $(72.4 \%, 85.7 \% ; 78 \%, 83 \%)$ and concluded that normal MCA PI is helpful to identify foetuses without major adverse perinatal outcome. ${ }^{30}$

\section{CONCLUSION}

Doppler patterns follow a longitudinal trend with early changes in the umbilical artery followed by middle cerebral artery and other peripheral arteries. Compared to other methods of foetal monitoring Doppler has proved to be more sensitive in detecting foetal compromises early and aids in the appropriate timing of delivery. In our study, MCA PI was the most sensitive and specific indicator for extent of foetal compromise in IUGR, though ratios such as MCA PI/UA PI as well as UA SD (>3) were comparably specific predictors for poor perinatal outcome. Hence, if UA PI is abnormal MCA PI should be performed to know extent of brain sparing, thus emphasizing importance of studying two vessels in Doppler. Non stress test can be used as an aid to Doppler findings in a compromised foetus as it is also non invasive and with possibility of in house monitoring, however it cannot be relied upon as a sole predictor of perinatal outcome.

Funding: No funding sources

Conflict of interest: None declared

Ethical approval: The study was approved by the Institutional Ethics Committee

\section{REFERENCES}

1. Sheridan C. Intrauterine growth restriction. Australian Family Physician. 2005;34(9):717-23.

2. Morris RK, Malin G, Robson SC, Kleijnen J, Zamora J, Khan KS. Foetal umbilical artery Doppler to predict compromise of foetal/neonatal wellbeing in a high-risk population: systematic review and bivariate meta-analysis. Ultrasound Obstet Gynecol. 2011;37(2):135-42.

3. Coomarasamy A, Fisk N, Gee H, Robson S. The Investigation and Management of the Small for Gestational Age Foetus. Guideline No. 31, Nov 2002. RCOG Press: London, 2003.

4. Ott WJ. The diagnosis of altered foetal growth. Obstet Gynecol Clin North Am. 1988;15:237-63.

5. Gagnon R, Van Den HM. The use of foetal Doppler in obstetrics. J Obstet Gynaecol Can. 2003;25:60114.

6. Lakhkar BN, Rajagopal KV, Gourisankar PT. Doppler Prediction of Adverse Perinatal Outcome in PIH and IUGR. IJRI. 2006;16:1:109-16.

7. Haram K, Softeland E, Bukowski R. Intrauterine growth restriction. International Journal of Gynaecology and Obstetrics. 2006;93:5-12.

8. Australian Health Ministers' Advisory Council. Clinical practice guidelines: Antenatal care- Module 1. Canberra: Australian Government Department of
Health and Ageing; 2012. Available from: http://www.health.gov.au/antenatal.

9. Kurmanavicius J, Florio I, Wisser J. Reference resistance indices of the umbilical, foetal middle cerebral and uterine arteries at 24-42 weeks of gestation- Ultrasound in Obstetrics and Gynaecology. 1997.

10. Gramellini D, Folli MC, Raboni S, Vadora E, Merialdi A. Cerebral-umbilical Doppler ratio as predictor of adverseperinatal outcome. Obstet Gynecol. 1992;79:416-20.

11. Mari G, Deter RL. Middle cerebral artery flow velocity waveforms in normal and small for gestational age foetuses-American journal of obstetrics and gynecology. 1992;166:1262-70.

12. Chauhan R, Samiksha Trivedi. Role of Doppler study in high risk pregnancy - Journal of obstetrics and gynaecology of India. 2002;529(3).

13. Acharya G, Wilsgaard T, Berntsen GKR, Maltau M, Kiserud T. Reference ranges for serial measurements of umbilical artery Doppler indices in second half of pregnancy. Am J Obstet Gynecol. 2005;192:937- 44.

14. Calvert JP, Crean EE, Newcombe RG, Pearson JF. Antenatal screening measurements by symphysis fundal height. BMJ [Clin Res]. 1982;285:846-9.

15. Doubilet PM, Benson CB. Sonographic evaluation of intrauterine growth retardation. AJR Am J Roentgenol. 1995;164:709-17.

16. Hadlock F. Ultrasound evaluation of foetal growth. In: Callen P, ed. Ultrasonography in obstetrics and gynaecology. $3^{\text {rd }}$ ed. Philadelphia: Saunders. 1994;129-42.

17. Benson CB, Doubilet PM, Saltzman DH, Jones TB. FL/AC ratio: poor predictor of intrauterine growth retardation Invest Radiol. 1985;20(7):727-30.

18. Bhimarao RM, Bhat NV, Gowda PVN. Efficacy of tcd/ac vs. Hc/ac in predicting asymmetric IUGR. Journal of Clinical and Diagnostic Research. 2015;9(10):TC01-5.

19. Eik-Nes SH, Salvesen KA, Okland O, Vatten LJ. Routine ultrasound foetal examination in pregnancy: the 'Alesund' randomized controlled trial. Ultrasound Obstet Gynecol. 2000;15:473-8.

20. Grover V, Usha R, Kalra S, Sachdeva S. Altered foetal growth: antenatal diagnosis by symphysisfundal height in India and comparison with Western charts. Int J Gynaecol Obstet. 1991;35:231-4.

21. Lindhard A, Nielsen PV, Mouritsen LA, Zachariassen A, Sorensen HU, Roseno H. The implications of introducing the symphyseal-fundal height-measurement. A prospective randomized controlled trial. Br J Obstet Gynaecol. 1990;97:67580 .

22. Morse K, Williams A, Gardosi J. Fetal growth screening by fundal height measurement. Best Pract Res Clin Obstet Gynaecol. 2009;23:809-18.

23. Gardosi J, Francis A. Controlled trial of fundal height measurement plotted on customized antenatal growth charts. Br J Obstet Gynaecol. 1999;106:30917. 
24. Royal College of Obstetricians and Gynaecologists. The investigation and management of the small-forgestational age foetus. RCOG Green Top Guideline no. 31. London; 2002. Available at: www.rcog.org.uk/files/rcogcorp/ Uploaded files/GT31SmallGestationalAge Fetus.pdf.

25. Turan OM, Turan S, Gungor S, Berg C, Moyano D, Gembruch U, et al. Progression of Doppler abnormalities in intrauterine growth restriction: Ultrasound Obstet Gynecol. 2008;32:160-7.

26. Gudmundsson S, Marsal K. Umbilical artery and uteroplacental blood flow velocity waveforms in normal pregnancy-a cross - sectional study - Acta Obstetrica Gynaecological Scandinavia; 1988:67.

27. Yoon BH, Lee CM, Kim SW. An abnormal umbilical artery waveform; a strong and independent predictor of adverse perinatal outcome in patients with pre eclampsia -American journal of obstetrics and gynaecology. 1994;171:713-21.

28. Bano S, Chaudhary V, Pande S, Mehta VL, Sharma AK. Colour Doppler evaluation of cerebral umbilical pulsatility ratio and its usefulness in the diagnosis of intrauterine growth retardation and prediction of adverse perinatal outcome. Indian J Radiol Imaging, 2010;20(1):20-5.

29. Rizzo G, Caponi A, Arduini D, Romanini C. The value of foetal arterial, cardiac and Venous flows in predicting ph and blood gases measured in umbilical blood at cordocentesis in growth retarded foetusesBritish journal of obstetrics and gynaecology. 1995;102:963-9.

30. Fong KW, Ohlsson A, Hanah Me, Kingdom J. Prediction of Perinatal Outcome in Foetuses Suspected to Have Intrauterine Growth Restriction. Doppler US Study of Foetal Cerebral, Renal, and Umbilical Arteries - Radiology. 1999;213:681-9.

Cite this article as: Sharma DD, Chandnani KC. Clinical study of IUGR cases and correlation of Doppler parameters with perinatal outcome. Int J Reprod Contracept Obstet Gynecol 2016;5:4290-6. 